\title{
Juan-Luis Klein*
}

\section{Iniciativa local y desarrollo: respuesta social a la globalización neoliberal $^{* *}$}

\begin{abstract}
This paper presents a synthesis of the local development and adresses the place of the local in the development theory. It is proposed that the territory role is essential in the development of a community. Territory is the ground for sens of belonging-based social arrangements of territorial actors. The paper is divided in four sections: a) the evolution of the "local" concept; b) the rise of local development; $c$ ) the approaches of local development; and d) a scheme that shows that local collective action and territory are conjugated through the territorial identity which is the basis for a local system structuration. It is proposed that local development programs should fertilize the territory, so that it becomes a launch pad for initiatives, projects and collective actions. For this, multiscale governance, a networking perspective and the interrelation among the local, the national and the world scales are required.
\end{abstract}

Keywords: Local, territory, collective action, identity, development, governance.

\section{Resumen}

Este texto presenta una síntesis del desarrollo local y de lo local en el desarrollo. En el desarrollo de una colectividad, el papel del territorio es fundamental. El territorio actúa como marco instituyente de arreglos sociales ligados a la pertenencia territorial de los actores. El texto se divide en cuatro secciones: a) la evolución del concepto de local; b) la emergencia del desarrollo local; c) los diversos enfoques; y d) proposición de un esquema que muestra cómo la acción local y el territorio se conjugan a través de la pertenencia territorial y de la identidad en un proceso de creación sistémica. Se propone que el desarrollo local debe fertilizar el territorio, de manera que este se transforme en una plataforma de lanzamiento de iniciativas, de proyectos y de acciones colectivas. Esto exige una gobernanza multi-escalar, una perspectiva reticular y la interrelación entre lo local, lo nacional y lo mundial.

Palabras clave: local, territorio, acción colectiva, identidad, desarrollo, gobernanza. 


\section{Introducción}

$\mathrm{L}$ a perspectiva del desarrollo local es el resultado de la voluntad de actores sociales, políticos y económicos de intervenir de manera activa en los procesos de desarrollo acaecientes en sus territorios y de influir en la reconversión de estos a la nueva economía, es decir a la economía del conocimiento. Más que a una teoría científica y rigurosa, el desarrollo local corresponde a una visión multidisciplinaria que incluye lo económico, lo político, lo social y evidentemente lo territorial. Esta visión instruye las formas recientes que toma la intervención en lo que respecta al ordenamiento y a la planificación territorial. "Los emprendimientos económicos no pueden florecer en un desierto social”, plantea Vachon ${ }^{1}$. Lo que sostendremos en este texto es que el desarrollo local comprende los métodos que permiten fertilizar territorios empobrecidos como resultado de relaciones de desarrollo desigual y de las crisis que provocan los cambios recientes en el mercado.

En el desarrollo de una colectividad, el papel del territorio es fundamental, en la medida que este genera identidad (Arocena, 2001), y que, como resultado de esta identidad, actores socialmente contrapuestos

* Centro de Investigación sobre las Innovaciones Sociales (CRISES); Departamento de Geografía de la Université du Québec à Montréal. E-mail: juan-luis.klein@uqam.ca.

** Este texto presenta una reflexión sintética a partir de una serie de trabajos empíricos realizados con los colegas J.M. Fontan, D.-G. Tremblay y B. Lévesque. Estos trabajos han recibido el apoyo del Consejo de Investigaciones en Ciencias Humanas de Canadá (CRSH). Las ideas contenidas en el texto han sido presentadas en dos eventos. El primero tuvo lugar en Córdoba, Argentina, y se tituló Las transformaciones de la modernidad excluyente y las politicas sociales, realizado con el auspicio de la Universidad Nacional de Córdoba (abril de 2005). El segundo se tituló Inégalités sociales et recomposition des territoires: de la mesure à l'intervention, realizado en la Universidad de Québec en Chicoutimi, en el marco del congreso anual de la ACFAS (mayo de 2005). Una versión bastante diferente de este texto, redactada con una perspectiva epistemológica, será publicada en un tratado de geografía humana que será publicado en México, dirigido por A. Lindón y D. HiernauxNicolás. Recibido el 19 de abril de 2005, aprobado el 30 de octubre de 2005.

${ }^{1}$ Citamos de memoria esta frase pronunciada frecuentemente por Bernard Vachon. Pour le point de vu de cet auteur, voir Vachon et Coallier (1993). se asocian y se estructuran en forma sistémica (Klein, 1997). Plantearemos en este texto que el desarrollo local deja como principal resultado la constitución de sistemas locales de actores. Dicho de otra manera, si el desarrollo local es local, esto no se debe a la localización del desarrollo en un lugar determinado. Si así fuera, toda forma de desarrollo sería local, puesto que toda acción se lleva a cabo en un lugar determinado. Las acciones de desarrollo pueden ser caracterizadas como desarrollo local cuando generan o refuerzan dinámicas sistémicas de escala local. A partir de esta perspectiva se ve el papel activo del territorio en tanto que marco instituyente de arreglos sociales, de estructuraciones sociales cuyo origen se debe a la pertenencia territorial de los actores.

En el desarrollo local convergen teorías y modelos que tienen orígenes disciplinarios distintos y que plantean métodos diversos, en ciertos casos contrapuestos. Lo que actúa como elemento unificador en el campo del desarrollo local son los cuestionamientos sobre los orígenes, los actores, las modalidades y los objetivos de las acciones de desarrollo. Para que se pueda hablar de desarrollo local, las acciones de desarrollo deben ser llevadas a cabo por actores locales (Gumuchian et al., 2003). En cuanto a sus objetivos, las acciones de desarrollo local deben habilitar a los actores locales con el fin de que estos puedan jugar un papel activo en el desarrollo de sus colectividades, que pueden llevar a cabo iniciativas y proyectos y de esta manera, movilizar recursos endógenos y exógenos ${ }^{2}$ en beneficio de la colectividad local (Arocena, 2001; Klein y Fontan, 2003).

El análisis que presentaremos del desarrollo local se divide en cuatro secciones. En primer lugar, abordaremos la evolución del concepto de local. En esta sección se mostrará que a pesar de que la noción de local tiene una importancia fundamental en los orígenes del análisis territorial en ciencias sociales, esta fue ocultada durante varias décadas como consecuencias de la generalización de las perspectivas keynesianas y del concepto de desarrollo regional. Lo local se redescubre cuando el modelo de crecimiento fordista y las modalidades keynesianas de

${ }^{2}$ Lo que incluye por cierto a los aportes públicos. El Estado debe jugar un papel fundamental en la puesta en práctica de una estrategia de desarrollo local. En esto, compartimos plenamente el análisis de Amin (2005). 
regulación de lo social entran en crisis a fines de los '70. En segundo lugar, consideraremos la emergencia del desarrollo local en tanto que respuesta ascendiente a una teoría del desarrollo que se caracterizaba por su dinámica descendiente. En tercer lugar, revisaremos diversos enfoques acerca de la estructuración de sistemas locales y territoriales que sirven de referencia a las acciones tanto políticas como económicas de desarrollo local. Se verá en esta sección que las diversas formas de desarrollo local se insertan en un mundo económico globalizado y en un cuadro político en el cual las formas tradicionales de la acción pública y de la ciudadanía son cuestionadas, lo que plantea el problema de la gobernanza y de la democracia. En cuarto lugar, propondremos un esquema que muestra cómo la acción local y el territorio se conjugan a través de la pertenencia territorial y de la identidad en un proceso de creación sistémica.

\section{Lo local como referencia social: del pre- fordismo al post-fordismo}

Si la noción de desarrollo local es reciente, la discusión acerca del rol que juega lo local en la estructuración de la sociedad es antigua. Esta discusión presenta varias facetas. Un de ellas es la faceta científica. En este ámbito, a fines del siglo XIX y a principios del siglo XX, tiene lugar un debate importante provocado por la construcción de la sociedad moderna y la confrontación entre una concepción que da prioridad a la institucionalidad nacional, y otra que defiende las estructuras y los valores de base comunitaria: la oposición entre la Gesellschaft y la Gemainschaft de Tönnies en Alemania, y entre la sociología globalista durkheimiana y la geografía localista vidaliana en Francia ${ }^{3}$. A la crítica durkheimiana, Vidal de la Blache responde: "La geografía es la ciencia de los lugares (science des lieux) y no la de los hombres". Por lugar, Vidal entiende una región definida como una unidad territorial cuyos componentes físicos y humanos son específicos y distintivos, interelacionados a través de lo que el designa como "géneros de vida" (genres de vie).

Este tipo de debate no fue solamente científico. También se expresa en el marco de la planificación y de la intervención socio-territorial. Por ejemplo, plan-

\footnotetext{
${ }^{3}$ Sobre esta controversia ver Capel (1981).
}

teamientos de origen socialista o anarquista insisten en la importancia de la estructuración de colectividades territoriales donde se integre el medio de vida y el medio de trabajo. Impulsados por este objetivo, diversos autores proponen, a principios del siglo XX, estrategias territoriales de base local, entre las cuales se destaca la proposición del urbanista inglés E. Howard. Este propone la planificación de pequeñas ciudades llamadas Garden Cities, como respuesta a la acción destructiva de la industrialización y de su correspondiente urbanización. Las Garden Cities debían reunir las ventajas de la ciudad y del campo, situarse en las afueras de las grandes ciudades y permitir a la colectividad de trabajar y vivir en el mismo marco territorial. Se trataba de construir unidades territoriales de escala humana ${ }^{4}$.

Por lo demás, es en esta época en la cual el economista A. Marshall descubre la existencia de colectividades locales que conocen un gran dinamismo empresarial a pesar de no poseer las ventajas de las grandes empresas. Estas colectividades estaban compuestas por redes productivas de pequeñas empresas y por formas importantes de colaboración entre éstas y la sociedad local. Estas colectividades, en las cuales reina una "atmósfera industrial”, corresponden a lo que Marshall identifica como "distritos industriales”, concepto básico de las estrategias actuales de desarrollo local ${ }^{5}$.

El interés acerca de lo local como base de comprensión o de acción en el dominio territorial persiste hasta los años ' 30 , época a partir de la cual la solución a la crisis de sobreproducción impone la regulación fordista caracterizada por la gestión keynesiana de lo social y por las estrategias económicas de escala nacional ${ }^{6}$. El fordismo como modo de regulación representa el triunfo de la globalidad nacional por sobre la especificidad local. En este marco, toda referencia a lo local es vista como una reacción frente a la modernidad.

\footnotetext{
${ }^{4}$ Acerca de la influencia de las ideologías comunitaristas y socialistas utópicas sobre la planificación regional y urbana, ver Gore (1984).

${ }^{5}$ Acerca de las ideas de Marshall y de la noción de distrito industrial, ver Beccatini (1992).

${ }^{6}$ En un texto anterior hemos hecho una síntesis de los efectos geográficos de la regulación fordista y keynesiana. Ver Klein (1999).
} 
Entre los años ' 40 y' 70 , en el conjunto de las sociedades occidentales -lo que incluye por supuesto a América Latina- se vive un periodo de crecimiento marcado por la modernización de las estructuras sociales, por la implantación, con grados diversos, del Estado benefactor y por la homogeneización económica a escala nacional. El Estado se erige en el centro de la gobernanza. Es también en este periodo en el que emerge la noción de desarrollo y en el cual se elaboran las principales teorías de desarrollo regio$\mathrm{nal}^{7}$. El desarrollo se presenta, en primer lugar, como una estrategia de expansión del modo de vida de las sociedades de producción y de consumo de masa hacia los países considerados como sub-desarrollados. En esta línea, W.W. Rostow propone la aplicación de una estrategia etapista de "desarrollo económico" y de "desarrollo político" destinada a producir el "despegue económico" (take off), y a reproducir en los países "atrasados" la trayectoria seguida por los países "avanzados".

Por otra parte, inspirados explícitamente en la reflexión del economista F. Perroux, quien establece la relación entre los conceptos de desarrollo y de innovación, y también, aunque implícitamente, en el pensamiento rostowiano, autores como J. Friedmann y J. Boudeville proponen una estrategia polarizada de modernización regional, la cual conduce a la homogeneización del territorio nacional. Según esta estrategia, en la mayoría de los países existen polos de desarrollo modernos, las grandes ciudades, caracterizados por el crecimiento y por la innovación. Pero en torno a estos polos existen colectividades tradicionales, donde la economía es poco productiva y donde las estructuras sociales conservan sus rasgos tradicionales. El desarrollo regional se da entonces el objetivo de modernizar las estructuras productivas, las relaciones sociales y los modos de producción dominantes en las regiones consideradas como tradicionales a fin de ponerlas al mismo nivel de crecimiento que existe en las grandes ciudades. Como ya se ha expresado, en este aspecto el desarrollo regional es coherente con la estrategia fordista de uniformización social, política y económica del territorio nacional. Es el triunfo de la socie-

${ }^{7}$ Acerca de la elaboración de las principales teorías en materia de desarrollo regional, se puede consultar Friedmann y Alonso (1964); Friedmann y Weaver (1979). dad nacional sobre la especificidad local, lo cual tiene enormes consecuencias en lo que respecta al análisis del papel de lo local en materia de desarrollo.

Nace así una visión de la intervención territorial que se inspira en el paradigma de la planificación regional. Esta visión sienta las bases del ordenamiento del territorio cuyo objetivo principal es establecer la conformidad entre los diversos niveles territoriales que constituyen la sociedad nacional. Esto conduce tanto a las políticas de desarrollo como a los actores políticos y sociales a darle la espalda a lo local. "Después de la Segunda Guerra Mundial, las teorías dominantes en las ciencias sociales en los países occidentales, fuertemente influenciadas por Estados Unidos, atribuían un papel muy limitado e insignificante al concepto de lugar (place)", afirma Agnew (1991), agregando: "el imperialismo americano y el concepto de lugar se excluyen mutuamente" (p. 145).

A partir de la segunda mitad de los años '70, esta concepción del territorio y de la intervención territorial se confronta con un nuevo cambio paradigmático, en parte como un efecto de la globalización y también como resultado de la emergencia del paradigma accionalista de $\mathrm{A}$. Touraine, el cual impone la introducción del actor (le retour de l'acteur) en el análisis social. Las ciencias del desarrollo redescubren los lugares en la medida que en ellos se engendra la acción colectiva, las especificidades locales y regionales, la unicidad de lo social (Fontan, Klein y Tremblay, 2005). A través de lo local se ven trayectorias específicas de adaptación de las sociedades a los espacios supranacionales de la globalización. Los Estados nacionales, que encarnaban tanto la société de Durkheim como la Gesellschaft de Tönnies, y que constituían el marco de referencia de la modernización de Rostow o del desarrollo regional de Friedmann, se han ido sometiendo progresivamente a reglas e instituciones que operan en una escala global, las cuales imponen lógicas supranacionales a los territorios nacionales. El territorio nacional deja de ser la escala única de la regulación centrada en el Estado (Brenner, 2003). En territorios locales y regionales se hacen sentir efectos estructurantes tanto en lo que corresponde a los procesos de desarrollo como a los sistemas de actores que toman parte en estos procesos, lo que contribuye a recentrar las relaciones sociales en un nivel local. 


\section{De la perspectiva descendente a la perspectiva ascendente}

Como se dijo anteriormente, como consecuencia de la crisis de sobreproducción de 1929 y como resultado de la aplicación de los métodos keynesianos que aportaron una solución a esta crisis, el territorio nacional se transforma en el marco espacial de coherencia de toda la acción estatal en materia de desarrollo. Los gobiernos ponen en práctica diversas formas de acción con el fin de disminuir las disparidades interregionales y de aumentar los niveles de consumo en las regiones consideradas como más "retrasadas". Esta perspectiva combina objetivos económicos y sociales, como lo demuestra el celebre caso de la Tennessee Valley Authority, pieza importante del New Deal implantado en Estados Unidos como respuesta a la crisis. A partir de esto, el Estado nación se impone como el referente obligado de las teorías sobre el desarrollo económico y social, incluyendo los análisis críticos como los que F.E. Cardoso o S. Amin elaboran en el marco de la teoría de la dependencia y de la teoría del desarrollo desigual.

Esta perspectiva respondía a dos tipos de factores. Por una parte, los Estados tratan de neutralizar las reivindicaciones de las clases sociales más necesitadas, las cuales son vistas como una amenaza a la estabilidad social. Y por otra parte, la necesaria incorporación de las más amplias capas de la población a la sociedad de consumo, un aspecto fundamental de la solución keynesiana a la crisis de sobreproducción, lleva a los gobiernos a intervenir en las regiones más pobres. Se aplican entonces políticas de desconcentración tanto de la industria como de la administración estatal y de modernización de los sectores tradicionales. El desarrollo regional se integra así en la planificación nacional.

Las políticas de desarrollo regional constituyen el principal instrumento de modernización y de unificación de los distintos "géneros de vida" existentes en el territorio. Esto tiene por lo menos tres consecuencias importantes que conviene subrayar, a fin de entender el vuelco posterior hacia lo local y el cambio de perspectiva que este implica:

La valorización de los recursos se hace de una manera centralizada y el desarrollo regional es controlado casi exclusivamente por los minis- terios, las empresas estatales y las sedes sociales de las grandes empresas (“centración” de las relaciones sociales en torno al Estado).

- Las políticas de desarrollo toman un sentido sectorial (forestal, industrial, tecnológico, etc.) y no territorial, favoreciéndose una integración espacial vertical únicamente en un nivel nacional (regulación fordista).

- El modo de vida propio del fordismo se difunde a través del territorio favoreciéndose la concentración de la población en los principales centros urbanos, a expensas de las culturas locales y de las formas no urbanas de ocupación del territorio (modernización).

El crecimiento que caracteriza la sociedad occidental entre los años ' 40 y '60 le da legitimidad a este modelo. Sin embargo, desde principios de los años '70 se comienza a advertir los primeros signos de una crisis que explota a fines de la década. Numerosos ejemplos son invocados en diversos países como testimonio de la ineficiencia de este modelo, y sobre todo de su incapacidad para lograr su principal objetivo, el equilibrio territorial y la equidad en la repartición territorial de la riqueza (Stöhr, 2003). Se denuncia el efecto perverso de esta política en lo que respecta a las crecientes desigualdades entre las principales ciudades y las regiones como resultado de la concentración de las inversiones públicas en lo que se consideraba como los polos de desarrollo. Se denuncia la centralización del aparato estatal y su efecto aniquilador de la capacidad de acción de los actores locales (Stöhr y Taylor, 1981; Laurelli y Rofman, 1989; Boisier, 1990). Como efecto de la crítica de un modelo considerado como estatista y centralizador, emergen fórmulas alternativas tales como el "autodesarrollo", el "desarrollo endógeno", el "desarrollo autocentrado", el "desarrollo integrado", etc. (Tremblay y Fontan, 1997).

Con ciertas diferencias de matices, todas estas fórmulas señalan un cambio de perspectiva. La perspectiva ascendente se presenta como la alternativa frente a la perspectiva descendente (Stöhr, 2003). El espacio local, visto en todas sus configuraciones en tanto que región, aglomeración y/o barrio o distrito urbano- es considerado la base a partir de la cual se generan iniciativas y proyectos de desarrollo 
económico que movilizan a la sociedad civil. La evidencia de la crisis del fordismo le da credibilidad y validez a esta nueva visión del desarrollo, que se denominará, a partir de entonces "desarrollo local" (Aydalot, 1983), en la cual priman lo local y la socie$\mathrm{dad}_{\mathrm{civil}}{ }^{8}$, sin que esto signifique que lo local y a los procesos de descentralización constituyan una panacea (De Mattos, 1988).

Esta nueva visión no se apoya en un modelo claro, lo que permite que refiriéndose al desarrollo local se planteen estrategias contrapuestas. Por un lado, la ortodoxia neoliberal -la cual, hay que subrayarlo, prima en la conducta de la sociedad globalizada y de los Estados a partir de los años '80- ve en el desarrollo local la posibilidad de deshacerse de ciertas responsabilidades sociales que tienen un coste financiero importante. En este marco, el desarrollo local se plantea como el traspaso de responsabilidades sociales a actores de la sociedad civil tales como instituciones locales, organizaciones de beneficencia, grupos de presión, que pueden asumirlas de manera más eficiente, y sobre todo, con recursos inferiores (Amin, 2005). Por otra parte, una perspectiva comunitaria plantea un cambio en la visión del Estado con el fin de configurar nuevos espacios de autonomía donde puedan afirmarse nuevas solidaridades locales, formas activas de identidad que les den al pueblo, localmente organizado, un mayor poder de participación en la toma de decisiones (Teisserenc, 1994; Tremblay y Fontan, 1997). Es lo que se ha designado como el empoderamiento o empowerment (Friedmann, 1992).

Las estrategias divergen, pero hay algo que es común a todos los puntos de vista. Es lo que se refiere al papel que se le atribuye al Estado en las acciones de desarrollo. Ya no se ve al Estado como el único actor del desarrollo, sino que como un socio (partner). La gestión centralizada del desarrollo es remplazada por una gestión mixta en la cual convergen el Estado y los actores locales. La asociación reemplaza a la confrontación y se crean instancias intermedias de concertación, cuya función es la armo-

\footnotetext{
${ }^{8}$ Joyal (2002) establece que el uso de la noción de desarrollo local es el resultado de la influencia francesa. Esta noción se contrapone a una opción conceptual de orige estadounidense: la community-based economic development.
}

nización de los intereses de los actores sociales en un nivel local y regional (Klein, 1992).

La perspectiva del desarrollo ascendente recibe un apoyo fundamental a comienzo de los años ' 80 . Diversos autores demuestran la existencia de sistemas territoriales en los cuales priman mecanismos locales de regulación y formas territoriales de innovación, lanzando así la tesis de los medios innovadores (Aydalot, 1986). En medio de la crisis del fordismo puesta de manifiesto por las quiebras empresariales y por el aumento del paro en las grandes regiones industriales de América del Norte y Europa, ciertos lugares semirrurales mostraban características diferentes. Los trabajos fundadores sobre este tipo de lugares son los que tratan acerca de la "tercera Italia" (Beccatini, 1992), es decir aquellas regiones italianas que no corresponden ni a la parte industrializada de este país (Milán o Roma) ni a su parte rural (el Mezzoggiorno) (Lévesque et al., 1995). Estos lugares son designados como "distritos industriales".

Los trabajos de estos autores se repercuten en diversos lugares del mundo, recibiendo un impulso fundamental cuando Piore y Sabel (1984) presentan esta forma de organización territorial de las empresas y de la colectividad como un modo de producción destinado a remplazar al fordismo. La perspectiva se amplía y diversos autores detectan características similares a las de los distritos industriales italianos tanto en los tecnopolos y parques tecnológicos alejados de las grandes metrópolis industriales como en vastas regiones donde reina la pequeña y mediana empresa, o en barrios y zonas de grandes ciudades industriales donde se concentran ciertos sectores productivos.

La principal característica de todos los casos observados es la existencia de formas de colaboración entre los actores, las cuales remplazan a las relaciones de confrontación que dominaron durante el fordismo. La colaboración se manifiesta de diversas maneras. En lugar de la gran empresa aislada del medio social se ven redes de empresas integradas en la colectividad. La sinergia y la colaboración tecnológica remplazan al aislacionismo clásico del sector industrial con respecto a los centros de investigación y de formación. La concertación estratégica orienta la acción de las élites dirigentes. Convenciones locales entre actores matizan la aplicación de normas nacio- 
nales en materia de salarios y de gestión de la mano de obra. Todo esto contribuye a delinear las características de lo que Benko y Lipietz (1992) llaman las "regiones ganadoras".

La regiones y lugares donde se concentra el desarrollo, con sus características y sus especificidades, traen a la memoria de diversos geógrafos y cientistas sociales la región y el lugar definidos por Vidal de la Blache, como lo subrayan Benko y Lipietz (1992). ¿Puede ser interpretado esto como una suerte de revancha histórica de un género de vida tradicional y holístico frente a la modernidad funcional y desestructurante? Por cierto que no. El marco local del cual hablamos es completamente diferente de aquel que abordaban Vidal de la Blache y sus discípulos. Aunque la memoria histórica y la identidad jueguen un papel mayor en su estructuración, este no es el resultado del pasado. Lo local del postfordismo se estructura en tanto que respuesta socioterritorial a la globalización económica (Fontan, Klein y Lévesque, 2003), y no como la sobrevivencia de sistemas sociales tradicionales que emergerían luego del retiro del fordismo. Como lo demuestran Gumuchian et al. (2003), Joyal (2002) y Arocena (2001), lo local del post-fordismo corresponde a sistemas de actores sociales, políticos y económicos; a sistemas de acción, como dice Teisserenc (1994), y es de esta manera que hay que analizarlo.

\section{El efecto sistémico de la territorialidad local}

Diversos enfoques se han elaborado con el objeto de explicar o de tipificar este tipo de lugares (regiones, zonas, ciudades, barrios, etc.), donde el territorio local contribuye a establecer lazos entre actores socio-económicos y a instituir sistemas territoriales. Varios de estos enfoques han sido instrumentalizados por políticas de desarrollo local, dándole prioridad a factores diferentes. Observaremos de manera somera las principales proposiciones de los enfoques que nos parecen más importantes: el enfoque productivo, el de la innovación, el político y el social.

\subsection{El enfoque productivo}

Lo que llamamos enfoque productivo persigue la puesta en práctica de "sistemas productivos loca- les", los cuales corresponden a configuraciones productivas basadas en la integración local de empresas y de actores socio-políticos (Lévesque et al., 1995). Varios términos han sido utilizados para designar estas configuraciones productivas, entre los cuales quizás el más conocido es el de "distrito". Como ya lo hemos dicho, este concepto fue utilizado por Beccatini (1992) para explicar el dinamismo económico de la llamada "tercera Italia". Beccatini ve en la colaboración entre las empresas, y en la colaboración entre éstas y la colectividad, un parecido con los "distritos industriales" detectados por Marshall.

El concepto de sistema productivo local se integra en una corriente teórica inspirada en la Escuela de la Regulación (Benko y Lipietz, 2000). Esta corriente ha dado lugar a trabajos mayores acerca del papel del territorio en las reestructuraciones del espacio fordista (Storper y Scott, 1989), de las "regiones ganadoras" (Benko y Lipietz, 1992) y de las convenciones que explican las variantes del desarrollo industrial (Salais y Storper, 1993).

Las proposiciones estratégicas planteadas sobre la base del concepto de sistema productivo local plantean la necesidad de reagrupar geográficamente a empresas y actores de una misma rama, a fin de producir una dinámica de desarrollo local. La premisa principal de estos trabajos sostiene que la proximidad espacial conduce a los actores socio-económicos a valorar la identidad territorial, y consecuentemente, a adoptar estrategias de gobernanza local con el fin de unificar la acción de los actores productivos y de las empresas, produciendo así las condiciones para el establecimiento de clusters productivos.

Estos trabajos sostienen que existe una relación entre proximidad espacial, innovación y dinamismo socio-económico en el contexto de la economía globalizada. Sin embargo, se ha demostrado también que esta relación no es automática; que para producir dinamismo económico, la proximidad espacial debe combinarse con otras formas de proximidad de tipo relacional (Grossetti, 2003; Dupuy y de Burmeistrer, 2003). También se entiende hoy en día, luego de diversos estudios de caso y de diversos análisis críticos -entre los cuales se distinguen los de May (1986) y Markusen (1996)-, que los sistemas productivos locales no se pueden analizar aislados de los otros componentes del espacio económico, y 
sobre todo, sin tener en cuenta su necesaria integración con las redes globales metropolitanas que teje la economía mundial (Scott, 2001; Klein, Tremblay y Fontan, 2003).

\subsection{El enfoque politico}

Lo que llamamos enfoque político tiene su punto de partida en una visión de la economía política construida a partir del estudio del lugar (the political economy of place) en medios urbanos estadounidenses (Logan y Molotch, 1987). El concepto de base de este enfoque es el de "coaliciones locales de crecimiento" (growth coalitions), las cuales permiten la distinción de diferentes regímenes urbanos.

Este enfoque, que pone en el centro del análisis el problema del poder -en particular del poder local- en el marco de la globalización (Stone, 1989), emerge en Estados Unidos en los años '80 y ha tenido gran influencia en los estudios urbanos (Booth y Jouve, 2005). El objetivo de los trabajos inspirados en él es el de comprender las reestructuraciones urbanas que se iniciaron en esa época en respuesta a la crisis de la base económica de las grandes ciudades industriales. El concepto de "coalición" es definido como el conjunto de redes formales e informales estructuradas entre los actores públicos y privados de una entidad territorial (Stone, 1989). La hipótesis principal de esta corriente plantea que las orientaciones y la eficiencia de las reestructuraciones puestas en práctica en las economías urbanas dependen del tipo y de la estabilidad de la coalición entre los actores socio-económicos que las llevan a cabo.

A partir de esta corriente, el concepto de coalición se puede definir como una configuración específica de actores susceptible de movilizar recursos internos y externos. Esta definición ha sido refinada por Kantor, Savitch y Haddock (1997), quienes proponen una tipología basada en tres criterios: la posición con respecto al mercado, las interrelaciones de los actores de la economía privada con las instituciones de gobierno y la participación ciudadana en la vida democrática. La aplicación de esta tipología establece que lo que domina en los procesos de desarrollo urbano en Estados Unidos es el corporatismo y el elitismo (Deitrick, 1999), de lo que se desprende la necesidad de instaurar coaliciones amplias e incluyentes ${ }^{9}$.

\subsection{El enfoque de la innovación}

Lo que llamamos enfoque de la innovación corresponde a trabajos que abordan el desarrollo local a partir del paradigma de la innovación (Alter, 2000). Este paradigma pone el acento en la importancia del conocimiento en la puesta en marcha de procesos innovadores. El conocimiento necesario para poner en práctica una innovación se construye colectivamente en un contexto reticular que incluye a productores y consumidores, a partir de una idea nueva que cambia las maneras de producir y de consumir de una colectividad y que la reconstruye en tanto que actor (Callon, 1989).

Diversos autores han demostrado que la innovación y el dinamismo económico y social se producen en el marco de sistemas territoriales de innovación donde se combinan en interacción empresas privadas, instituciones públicas y centros de investigación científica de alto nivel. Este tipo de colaboración genera conocimiento y permite la valorización local del conocimiento así producido (Holbrook y Wolfe, 2002).

Las colectividades locales deben confrontarse con el problema del aprendizaje (Wolfe, 2002). Se desprende de esto que el conocimiento no es solamente científico sino que también social, lo que plantea la necesidad de organizaciones de producción del conocimiento en interrelación con los actores sociales. La innovación tecnológica y la amplitud de su difusión dependen del nivel de estabilidad social y de equidad existente en una colectividad territorial (Braczyk, Cooke y Heidenreich, 1998). En esta relación juegan un papel fundamental el aprendizaje colectivo y su sedimentación en organizaciones que permiten construir sistemas de innovación de manera ascendente.

Trabajos recientes han demostrado que, siendo crucial, la innovación tecnológica no es una solución idílica, que tiene también efectos negativos a diversas escalas y que en sí, no basta para estructurar territorios innovadores (Hiernaux-Nicolás, 1999). Es fundamental reintroducir lo social en el análisis de la innovación (Klein y Fontan, 2004; Hillier, Moulaert

\footnotetext{
${ }^{9}$ Como en el caso de Barcelona. Ver Borja y Castells
} (1997). 
y Nussbaumer, 2004). El rol de las instancias públicas y los centros de investigación y de enseñanza, así como de las organizaciones públicas y socio-económicas es crucial en la gestación y en la difusión de las innovaciones tecnológicas. La estructuración de interrelaciones regionales y locales entre estos actores constituye la esencia de la "nueva trinidad" sobre la cual yacen los sistemas regionales de innovación (Storper, 1997).

\subsection{El enfoque social}

Lo que llamamos enfoque social se basa en los trabajos que postulan que el territorio local es un marco generador de lazos sociales y de acción colectiva (Denieuil, 1997) en relación con los movimientos sociales (Klein, 1996). Según esta perspectiva, el sentimiento de pertenencia territorial crea espacios comunitarios adaptados a la sociedad moderna, diversificados e insertos de diversas formas en la sociedad global. Esto significa, en cierta manera, que la oposición sociedad-comunidad está sobrepasada, puesto que las comunidades actuales no tienen la mismas características descritas por Tönnies (Laville, 1997). También los movimientos sociales se reterritorializan, desarrollando acciones frente a oponentes locales, nacionales o incluso de nivel internacional, acerca de problemáticas locales (Klein, Tremblay y Dionne, 1997). La referencia local de los actores, es decir su identidad territorial, les lleva a realizar acciones colectivas con objetivos económicos inspiradas por la pertenencia a un territorio local, reconciliando así la economía y la sociedad (Sainsaulieu, 1997).

De esta manera, emerge un enfoque económico que recibe denominaciones diferentes (economía social, solidaria, comunitaria, popular), dependiendo de la realidad concreta de la que se trate (Favreau, 2005). Estas acepciones indican la existencia de matices importantes: la realidad económica, social e institucional no es la misma en Buenos Aires, Montreal, París, Dakar o Los Angeles, aunque el modo de vida urbano y la metropolización conlleven un cierto isomorfismo. Pero en todos los casos se hace referencia a formas de producción de la riqueza en la que participa directamente la colectividad utilizando modalidades asociativas, tanto en la producción de bienes y/o de servicios como en sus beneficios, y movilizando recursos ligados al capital socio-territo- rial de una colectividad local (Fontan y Klein, 2004). Como lo observamos en un trabajo de investigación en vías de realización, la economía social y el territorio se definen y se redefinen recíprocamente y de manera progresiva (Bouchard et al., 2005).

La transformación del movimiento social ligada a la acción colectiva de naturaleza económica tiene efectos tanto en lo que se refiere a la confrontación entre los actores sociales y sus oponentes, como en lo relativo a las formas que toma la acción colectiva. En ellas intervienen medios de alto nivel tecnológico, como la utilización de Internet y el acceso a amplias redes de información y de comunicación, como lo muestran los ejemplos del movimiento zapatista en México y de las comunidades vecinales (asambleas y foros) en Buenos Aires durante el "cacerolazo" (Calello et al., 2004). También se actúa en el marco de la producción tecnológica y de la inversión en empresas y emprendimientos cuyo objetivo es el mercado global. Pero la inserción en lo global no limita ni elimina el anclaje local de estas acciones.

\subsection{La convergencia de lo productivo, lo político, lo tecnológico y lo social en la definición estratégica de lo local}

A pesar de sus diferencias, estos enfoques convergen en dos puntos. Por una parte, lo local no se puede definir en sí, sino que en su interrelación con redes globales. Por lo tanto, la delimitación de lo local es dinámica y no estática (ni estadística). Por otra parte, lo que acerca a los actores locales a pesar de la distancia social que puede separarlos, es su identidad territorial común, identidad por cierto exacerbada por la relación conflictiva con lo global (Joyal, 2002).

La noción misma de local no puede ser definida de una manera precisa. Por cierto, lo local está ligado con actores y sistemas locales, salvo que lo que es local para ciertos actores no lo es para otros. Las delimitaciones de lo local no son claras ni tampoco homogéneas para todos los actores de una colectividad. De esta manera, la colectividad local aparece como una base a partir de la cual se estructuran acciones; como una base de iniciativas tomadas por actores locales, de proyectos colectivos o individuales, los cuales, luego de una adhesión más amplia, se transforman en "acciones colec- 
tivas". La proximidad física, puesta de manifiesto por la identidad común, se transforma progresivamente en proximidad social.

Es importante concentrarse sobre la interrelación iniciativa/colectividad. Como hemos dicho, lo local no corresponde a un lugar determinado, sino que a un sistema de actores que se conciertan porque tienen una identidad común con respecto a un territorio, una conciencia territorial que les lleva a asociarse y a realizar proyectos en asociatividad. Por cierto, la inserción del territorio en lo que se designa como "nueva economía" o "economía del saber" es fundamental. Pero las condiciones para el desarrollo de un territorio deben ser múltiples, y la sola interrelación entre los actores locales no es suficiente para transformar "regiones perdedoras" en "regiones ganadoras" (Côté, Klein y Proulx, 1995; Markusen, 1996; Benko y Lipietz, 2000; Amin, 2005).

\section{La iniciativa local y su efecto territorial: proposición de un esquema interpretativo y de acción}

En lo que respecta a la intevención territorial con el fin de producir el desarrollo, el desafío que se plantea es el de generar los factores que permiten o que provocan la innovación. Un medio innovador y dinámico es un medio socialmente construido, apto a la innovación y al cambio. Pero, ¿por dónde comenzar? Como hemos dicho, diversos autores insisten sobre la sinergia entre los actores a fin de movilizar las dotaciones internas de las colectividades locales con fines de creación de riqueza. Salvo que esto no puede fructificar sin el apoyo de inversiones externas, públicas y privadas, necesarias para apoyar la actividad económica local (Markusen, 1996), y sin medios que revitalicen a los actores socio-económi$\cos$ (Amin, 2005). Definitivamente, el desarrollo local no puede limitarse a lo endógeno. Como se ha mostrado a partir del caso de un tecnopolo construido como resultado de une experiencia local y comunitaria en la ciudad de Montreal (Klein, Fontan y Tremblay, 2003), su puesta en marcha necesita la combinación de recursos locales y exteriores, endógenos y exógenos. Por lo demás, lo que permite a una localidad o región reequilibrar sus relaciones con regiones o localidades más centrales es justamente su capacidad de movilizar recursos exteriores, inclu- yendo por cierto a los recursos del Estado. Si no, sólo se reproduce una relación de desigualdad (Côté, Klein y Proulx, 1995).

Por lo demás, la implicación de los actores sociales locales en el desarrollo no es automática. Se ha demostrado que ésta constituye el resultado de un proceso en que interviene la identidad territorial compartida y la relación conflictiva con lo global. También se ha demostrado que la importancia de la acción colectiva y de la movilización social, siendo local, va mucho más allá de lo local. Nuevas formas de acciones colectivas son puestas en práctica con el fin de conservar medios de vida y de trabajo. El desarrollo local no puede limitarse a una reacción social frente a la crisis, sino que debe plantearse como el inicio de un proceso cíclico que instaure una dinámica de desarrollo basado en la iniciativa local y en la movilización de activos endógenos y exógenos (Pecqueur, 1989).

Para interpretar el efecto de la acción colectiva sobre el desarrollo, proponemos un esquema que pone en juego diversos elementos que contribuyen a crear el medio local, que consideramos como una construcción social, en tanto que marco de referencia de los actores socio-económicos (figura 1). El esquema propuesto está basado en numerosos estudios de casos exitosos, pero también en el estudio de casos que no han tenido el éxito esperado o que han fracasado. Esto nos lleva a identificar las etapas de un ciclo que nos parece vital para poner en marcha un proceso de desarrollo local y que señala los momentos estratégicos en los cuales las organizaciones públicas, privadas y sociales deben intervenir a fin de apoyarlo.

La primera etapa de este ciclo es el lanzamiento de una iniciativa, es decir, de un proyecto individual o colectivo. Hablamos de diversos tipos de proyectos y no sólo de proyectos empresariales (valorización de un recurso cultural o humano, protección de algún aspecto del patrimonio colectivo natural o construido, creación de empleo en la colectividad local, etc.). El proyecto así concebido se confronta con otros proyectos posibles. Individual en su origen, en la medida que interpreta las necesidades locales el proyecto se transforma en un proyecto colectivo, en un objetivo social impulsado por los actores y grupos sociales que constituyen la colectividad lo- 
cal. En su defensa, los actores locales realizan acciones colectivas que tienden a mostrar la importancia del proyecto y a desarrollar el apego de la colectividad a su realización.

En esta etapa, los actores locales orientan su capacidad financiera y organizativa en torno a asegurar la viabilidad del proyecto, asociándose también con instituciones y organizaciones externas. La lucha por la realización del proyecto actúa como cemento entre los actores, creando entre ellos un sentimiento de solidaridad. La solidaridad orienta la acción de los actores locales, llevándoles a poner énfasis en los que los une y no en lo que los desune. Los actores desarrollan así una conciencia colectiva delimitada territorialmente, la cual constituye una base esencial de la acción conjunta de los actores locales sociales y económicos, una base de emprendimientos "partenariales". Esta base puede cristalizarse en instituciones que sedimentan la experiencia dejada por la acción colectiva y que a través del aprendizaje actúan como medio favorable al lanzamiento de nuevas iniciativas y proyectos, reproduciendo así el ciclo.

La premisa del esquema propuesto es que el desarrollo local no puede limitarse a los recursos locales. $\mathrm{Al}$ movilizar recursos privados y públicos, individuales y colectivos, la acción colectiva establece una relación ascendente entre lo local y lo global, entre lo social y lo económico. En el fondo, lo que importa no es tanto el origen de los recursos movilizados por los actores locales, como la dinámica social que permite que un conjunto de recursos pueda ser movilizado en beneficio de la comunidad local; es esta dinámica que las estrategias y las políticas de desarrollo local deben tratar de impulsar y encauzar.

\section{Conclusión}

Como se ha dicho, la referencia a lo local se impone en la planificación territorial y en el ordenamiento del territorio a partir de los años ' 80 , remplazando así al desarrollo regional centrado en el Estado. Lo que en su momento fue una perspectiva alternativa, una innovación, se ha transformado en un marco de referencia importante para las políticas públicas y para la intervención en lo que respecta al territorio ${ }^{10}$. Hay que decir, sin embargo, que el éxito del desarrollo local se manifiesta claramente en casos en los cuales la colectividad dispone de recursos abundantes, como en el caso de zonas, corredores o barrios específicos de grandes metrópolis (Amin, 2005). Su éxito ha sido menos evidente cuando se ha tratado de reencauzar rumbos y de producir dinamismo en regiones desheredadas, sobre todo en regiones rurales, como lo señala Polèse (1996). Es que no se le puede pedir a una región desvitalizada que actúe

${ }^{10}$ Como lo demuestra el libro Manual de desarrollo local, publicado bajo la dirección del geógrafo Fermín Rodríguez (1999).

Figura 1. Desarrollo e iniciativa local: un esquema.

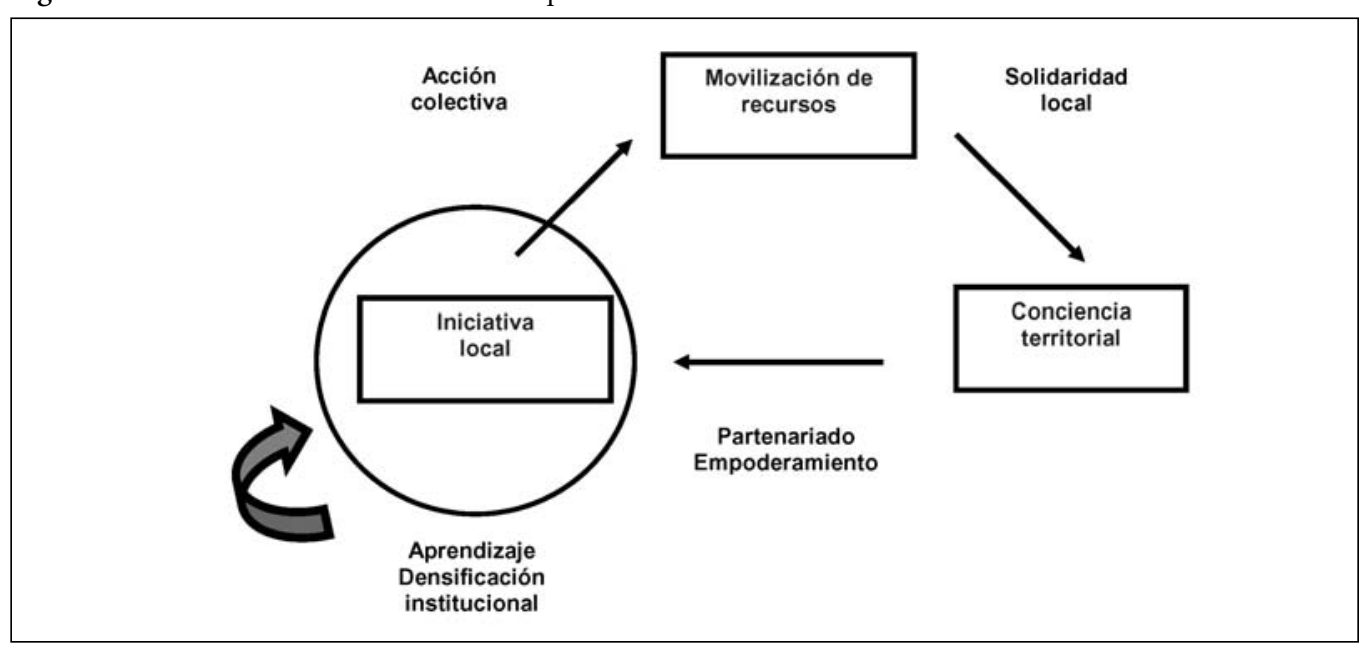


como una región dinámica y como un medio innovador cuando la causa de su desvitalización es justamente la ausencia en ella de los factores necesarios para generar dinamismo e innovación, y cuando lo que reina es el ciclo de la desvitalización.

La institucionalización del desarrollo local le ha dado a éste un cariz técnico. Por ejemplo, en Francia se han "labelizado" los sistemas de producción local (la "fórmula SPL"), y hoy en día, los casos de comunas asociadas con el fin de hacerse reconocer como SPL son múltiples (Pommier, 2002). El rol de agente de desarrollo local se ha profesionalizado, y lo que en un principio fue una perspectiva anclada en la base tiende a transformarse en una receta tecnocrática (OCDE, 2001). Por la misma razón de su institucionalización, el desarrollo local ha perdido algunos de los principios que constituían su base. Los objetivos de justicia y de equidad son a menudo relegados por la idea del empoderamiento de regiones y lugares con el fin de que éstas puedan competir con éxito en una economía abierta. Esto tiene como resultado la exacerbación de la competencia entre colectividades locales por atraer a recursos públicos o privados cada vez más escasos.

Existe consenso sobre el hecho de que el desarrollo a partir de la iniciativa local debe plantearse sobre la base del desarrollo durable, el cual debe verse tanto en su dimensión temporal como geográfica. La dimensión temporal del desarrollo durable está clara y bien aceptada. Se trata de que el desarrollo actual de una colectividad no se haga a expensas del potencial de desarrollo de las generaciones futuras. Pero también habría que agregar que el desarrollo de una colectividad no debería hacerse a expensas del desarrollo de otras colectividades. Este aspecto está menos claro, puesto que se ven frecuentemente casos de competencia entre colectividades locales de un mismo país o de países diferentes por la realización de un proyecto o por la atracción de una determinada inversión pública o privada, lo que produce regiones ganadoras y perdedoras. Es necesario entonces sobrepasar el localismo y ver el desarrollo local con una perspectiva global.

A pesar de estas reservas, en el contexto del postfordismo, el territorio local permite reconstruir lazos sociales y relaciones de solidaridad. Esto, sin embargo, no es automático. La construcción de la solidaridad social a partir de la base es un proyecto social más que una realidad, como lo dice Piole (1990). Se trata entonces, como lo sostiene el esquema que hemos propuesto, de fertilizar el territorio, actuando en los momentos estratégicos, de manera que el territorio se transforme en una plataforma de lanzamiento de iniciativas, de proyectos y de acciones colectivas. Se trata de la reconstrucción de lo social a partir de la base, a partir de acciones colectivas que despierten o intensifiquen la conciencia territorial. Esto exige una opción territorial que incluya una gobernanza multi-escalar, una perspectiva reticular y la interrelación entre lo local, lo nacional y lo mundial.

Es necesario poner en práctica modalidades estratégicas de gobernanza amplias e incluyentes, adecuadas al cambio y a la conversión económica, que articulen lo productivo y lo social en una perspectiva de economía social y solidaria (Fontan, Klein y Lévesque, 2003). En lo territorial, esto se traduce en una visión estratégica pluralista donde tienen que impulsarse y combinarse tanto las inciativas del capital privado y de las instancias públicas, como aquellas que emergen a partir de la sociedad civil, así como los puentes necesarios para establecer lazos entre ellas. Así planteado, el desafío es elaborar una visión multifacética en la cual lo local permita recrear lo global, imbricando las diversas escalas del desarrollo, y en la cual el desarrollo no se haga a expensas de la colectividad, sino que en su beneficio.

\section{Referencias bibliográficas}

Agnew, J. (1991). "Les lieux contre la sociologie politique”. Lévy, J. (ed.) Géographies du politique. Paris: Presses de la fondation nationale des sciences politiques: 145-160.

Alter, N. (2000). Linnovation ordinaire. Paris: PUF. Amin, A. (2005). "Local community on trial". Economy and Society (À paraître).

Arocena, J. (2001). El desarrollo local: un desafio contemporáneo. Montevideo: Taurus.

Aydalot, P. (1983). "Réalités et illusions de l'économie locale". Autrement, 47, 83: 208-216. (ed.) (1986). Milieux innovateurs en Europe. Paris: Groupe de recherche européen sur les milieux innovateurs (GREMI).

Beccatini, G. (1992). "Le district marshallien: une notion socio-économique”. Benko, G. y A. 
Lipietz (eds.), Les Régions qui gangent. Districts et réseaux: les nouveaux paradigmes de la géographie économique. Paris, PUF: 35-55.

Benko, G. y A. Lipietz (eds.) (1992). Les régions qui gagnent, districts et réseaux: les nouveaux paradigmes de la géographie économique. Paris: PUF. (eds.) (2000). La richesse des régions. Paris: PUF.

Boisier, S. (1990). Territorio, Estado y sociedad. Valparaíso: Pehuén.

Borja, J. y M. Castells (1997). Local \& global: Management of cities in the information age. London: Earthscan Publications.

Booth, P. y B. Jouve (dirs). (2005). Metropolitan democraties. Transformations of the State and urban policy in Canada, France and Great Britain. Hampshire: Ashgate.

Bouchard, M., J. Carrière, J.-L. Klein, S. Marceau, V. Michaud y C. Saucier. (2005) "Les repères territoriaux de l'économie sociale et solidaire". Texto de una ponencia presentada en Marsella en el coloquio Economie sociale et solidaire et territoire, mayo.

Braczyk, H., P. Cooke y M. Heidenreich (eds.) (1998). Regional innovation systems. London: UCL Press.

Brenner, N. (2003). "La formación de la ciudad global y el re-escalamiento del espacio del Estado en la Europa Occidental post-fordista”. EURE Revista Latinoamericana de Estudios Urbano Regionales, 29, 86: 5-25.

Calello, T. F. Fritzche, A. Quintar y M. Vio (2004). "Redes y nuevas tecnologías de información y comunicación en las asambleas vecinales de Buenos Aires". Economía Sociedad Territorio, 4, 16: 609-633.

Callon, M. (1989). Innovation et ressources locales. Paris: PUF.

Capel, H. (1981). Filosofía y ciencia en la geografía contemporánea. Una introducción a la geografía. Barcelona: Barcanova.

Côté, S., J.-L. Klein y M.-U. Proulx (eds.) (1995). Et les régions qui perdent? Rimouski: GRIDEQ.

De Mattos, C. (1988). La descentralización, ¿una nueva panacea para impulsar el desarrollo local? Santiago: ILPES, Serie Ensayo.

Deitrick, S. (1999). "The Post Industrial Revitalization of Pittsburgh: Myths and Evidence". Community Development Journal, 34: 1: 4-12.
Denieuil, P.-N. (1997). Lien social et développement économique. Paris: L'Harmattan.

Dupuy C. y A. de Burmeistrer (eds.) (2003) Entreprises et territoires, les nouveaux enjeux de la proximité. Paris: Documentation française.

Favreau, L. (2005). "Économie sociale et développement local au Nord et au Sud; essai de comparaison”. Klein, J.-L. y C. Tardif (eds.), Entre réseaux et systèmes: les nouveaux espaces régionaux. Rimouski: GRIDEQ (en prensa).

Fontan, J.-M. y J.-L. Klein (2004). "La mobilisation du capital socio-territorial: le cas du technopôle Angus". Lien social et Politiques-RIAC, 52: 139149.

Fontan, J.-M., J.-L. Klein y B. Lévesque (2003). Reconversion économique et développement territorial: le rôle de la société civile. Québec: Presses de l'Université du Québec.

Fontan, J.-M., J.-L. Klein y D.-G. Tremblay (2005). Innovation socioterritoriale et reconversion économique: le cas de Montréal. Paris: L'Harmattan.

Friedmann, J. y W. Alonso (eds.) (1964). Regional development and planning. Boston: The M.I.T. Press.

Friedmann, J. y C. Weaver (1979). Territory and function. The evolution of regional planning. Berkeley: University of California Press.

Friedmann, J. (1992). Empowerment, the policy of alternative development. Cambridge: Blackwell.

Gore, C. (1984). Regions in question. Space. Development theory and regional policy. London/ New York: Methuen.

Grossetti, M. (2003). "Développement urbain technoscientifique”. Fontan, J.-M., J.-L. Klein y B. Lévesque (eds.), Reconversion économique et développement territorial: le rôle de la société civile. Québec: Presses de l'Université du Québec, 161182.

Gumuchian, H., E. Grasset, R. Lajarge y E. Roux (2003). Les acteurs, ces oubliés du territoire. Paris: Anthropos.

Hiernaux-Nicolás, D. (1999). Los senderos del cambio. Sociedad, tecnología y territorio en los albores del siglo XXI. México: Plaza Valdés.

Hillier, J., F. Moulaert y J. Nussbaumer (2004). “Trois essais sur le rôle de l'innovation sociale dans le développement territorial”. Géographie, Économie, Société, 6, 2: 129-152. 
Holbrook, J. y D. Wolfe (eds.) (2002). Knowledge, clusters and regional innovation. New York: McGill-Queens University Press.

Joyal, A. (2002). Le développement local. Comment stimuler l'économie des régions en difficulté. Québec: Éditions de l'IQRC.

Kantor, P., H.V. Savitch y S.V. Haddock (1997). "The political economy of urban regimes: A comparative perspective". Urban Affairs Review, 32, 3: 348-377.

Klein, J.-L. (1996). “Les mobilisations territorialisées et le développement local: vers un nouveau mouvement social?" Économie et Solidarité, 28, 1: 31-43.

(1992). "Le partenariat: vers une planification flexible du développement local?" Revue canadienne des sciences régionales, 15, 3: 491-505.

(1997). "L'espace local à l'heure de la globalisation: la part de la mobilisation sociale". Cahiers de géographie du Québec, 41, 114: $367-$ 377.

(1999). "Mondialisation et État-nation: la restructuration territoriale du système-monde". Klein, J.-L. y S. Laurin (eds.), L'Éducation géographique. Conscience territoriale et formation du citoyen. Sainte-Foy: Presses de l'Université du Québec, 55-92.

Klein, J.-L. y J.-M. Fontan (2003). “Reconversion économique et initiative locale: l'effet structurant des actions collectives". Fontan, J.M., J.-L. Klein y B. Lévesque (eds), Reconversion économique et développement territorial: le rôle de la société civile. Quebec: Presses de l’Université du Québec, 11-33.

(2004).

"Innovation sociale et territoire". Géographie, Économie, Société, 6, 2.

Klein, J,-L., P.-A. Tremblay y H. Dionne (eds.) (1997). Au delà du néolibéralisme: quel rôle pour les mouvements sociaux? Sainte-Foy: Presses de l'Université du Québec.

Klein, J.-L., D.-G. Tremblay y J.-M. Fontan (2003). "Systèmes locaux et réseaux productifs dans la reconversion économique: le cas de Montréal”. Géographie, Économie, Société, 5, 1:59-75.

Klein, J-L., J.-M. Fontan y D.-G. Tremblay (2003). "Reconversión y desarrollo a través de la iniciativa local: el caso de Montreal en Quebec". EURE
Revista Latinoamericana de Estudios Urbano Regionales, 29, 86: 69-88.

Laurelli, E. y A. Rofman (1989). Descentralización del Estado. Requerimientos y politicas en la crisis. Buenos Aires: CEUR.

Laville, J.-L. (1997). "Communauté, société et modernité”.Denieuil, P.-N. (ed.), Lien social et développementéconomique. Paris : L'Harmattan, 53-67.

Lévesque, B., J.-L. Klein, J.-M. Fontan y D. Bordeleau (1995). Systèmes locaux de production: Réflexion-synthèse sur les nouvelles modalités de développement régionalllocal. Montréal: Cahiers du CRISES, 9601.

Logan, J.R. y H.L. Molotch (1987). Urban fortunes. The political economy of place. Berkeley: University of California Press.

Markusen, A. (1996). "Sticky places in slippery space: A typology of industrial districts". Economic Geography, 72, 3: 293-313.

May, N. (1986). "Constitution d'un regard. Fordisme et localisme". Les annales de la recherche urbaine, 29: 5-12.

OCDE (2001). Des partenariats locaux pour une meilleure gouvernance. Paris: OCDE.

Pecqueur, B. (1989). Le développement économique local. Paris: Syros.

Piole, X. (1990). "Proximité géographique et lien social, de nouvelles formes de territorialité?" L'espace géographique, 19-20, 4: 349-358.

Piore, M. y C. Sabel (1984). The second industrial divide. New York: Basic Books.

Polèse, M. (1996). "Le développement local, revu et corrigé: récit d'une douce illusion dangereuse". Le Québec des régions: vers quel développement? Rimouski: GRIDEQ, 321-335.

Rodríguez, F. (dir.) (1999). Manual del desarrollo local. Gijón: Ediciones Trea S.L.

Pommier, P. (2002). "Les systèmes productifs locaux". Paris: Documentation Française, DATAR.

Sainsaulieu, R. (1997). "Changement des institutions et développement local". Denieuil, P.-N. (ed.), Lien social et développement économique. Paris: L'Harmattan, 9-13.

Salais, R.y M. Storper (1993). Les mondes de production. Paris: Éditions de l'EHESS.

Scott, A. (ed.) (2001). Global city-regions. Trends, theory, policy. Oxford: Oxford University Press. 
Stöhr, W. (2003). “Development from Below: Vingt ans plus tard”. Fontan, J.-M., J.-L. Klein y B. Lévesque (eds.), Reconversion économique et développement territorial: le rôle de la société civile. Québec: Presses de l'Université du Québec, 119143.

Stöhr, W. y F. Taylor (eds.) (1981). Development from above or below? The dialectics of regional planning in developing countries. Chichester: Wiley \& Sons Ltd.

Stone, C.S. (1989). Regime politics: Governing Atlanta (1946-1988). Lawrence: Kansas University Press.

Storper, M. y A. Scott (1989). “The geographical foundations and social regulation of flexible production complexes". Dear, M. y J. Wolch (eds), The power of geography. Boston: Unwin Hyman, 21-40.
Storper, M. (1997). "Les nouveaux dynamismes régionaux: conventions et systèmes d'acteurs". Coté, S., J.-L. Klein y M.-U. Proulx (dirs.), Action collective et décentralisation. Rimouski: GRIDEQ, 1-17.

Teisserenc, P. (1994). Les politiques de développement local. Paris: Economica.

Tremblay, D.-G. y J.-M. Fontan (1997). Le développement économique local. Les théories, les pratiques, les expériences. Sainte Foy: Presses de l'Université du Québec.

Wolfe, D. (2002). "Social capital and clusters development in learning regions". Holbrook, A. y D. Wolfe (eds.), Knowledge, clusters and regional innovation. Toronto-Montreal: McGillQueens University Press, 11-38. 